University of Nebraska - Lincoln

DigitalCommons@University of Nebraska - Lincoln

May 1993

\title{
SCALES: A Computer Program to Convert among Three Developmental Stage Scales for Wheat
}

\author{
Dorothy M. Harrell \\ University of Nebraska-Lincoln, dharrell2@unl.edu \\ Wallace Wilhelm \\ University of Nebraska-Lincoln, wwilhelm1@unl.edu \\ G. S. McMaster \\ USDA-ARS, greg.mcmaster@ars.usda.gov
}

Follow this and additional works at: https://digitalcommons.unl.edu/usdaarsfacpub

Part of the Agricultural Science Commons

Harrell, Dorothy M.; Wilhelm, Wallace; and McMaster, G. S., "SCALES: A Computer Program to Convert among Three Developmental Stage Scales for Wheat" (1993). Publications from USDA-ARS / UNL Faculty. 95.

https://digitalcommons.unl.edu/usdaarsfacpub/95

This Article is brought to you for free and open access by the U.S. Department of Agriculture: Agricultural Research Service, Lincoln, Nebraska at DigitalCommons@University of Nebraska - Lincoln. It has been accepted for inclusion in Publications from USDA-ARS / UNL Faculty by an authorized administrator of DigitalCommons@University of Nebraska - Lincoln. 


\title{
SCALES: A Computer Program to Convert among Three Developmental Stage Scales for Wheat
}

\author{
D. M. Harrell, ${ }^{*}$ W. W. Wilhelm, and G. S. McMaster
}

\begin{abstract}
The Haun, Feekes, and Zadoks-Chang-Konzak developmental stage scales are often used to report phenological information for wheat (Triticum aestivum L.) development. Agronomists familiar with one of these scales may have only a passing acquaintance with developmental scales other than the one they consider most appropriate for their purposes. This makes review and interpretation of the literature more difficult. Although wheat development models often report phenological results, they generally use no more than one scale. For these reasons, and because there is not a simple numeric correspondence among the three scales, a computer program to convert among them is a useful tool. SCALES is a three-module FORTRAN program that relates the three scales based on published descriptions. The interactive user interface module requests an input scale value and minimal additional information, which the conversion module uses to determine values for the remaining two scales via a dichotomous key. The output module writes the conversion results to the monitor. SCALES may be used independently for comparing phenological data based on different developmental scales, or the conversion module may be incorporated into computer models to provide output of developmental stage information for the simulation.
\end{abstract}

$\mathrm{D}$ EVELOPMENTAL STAGE SCALES are widely used to report the phenology of wheat. These scales provide an easily understood means of assessing developmental progress and are useful in comparing the effects of different management practices and environmental conditions. The timing of management practices, such as herbicide application, is often based on the stage of crop development. Agronomists use different scales, making the review and interpretation of the literature difficult because the reader must convert results to a familiar scale for comparison.

Wheat simulation models often incorporate at least part of existing phenological development stage scales into their programs. Scale values are helpful in evaluating the effects of changes in crop and environmental parameters. They may also be used in model validation and in comparing results of different models. Attaching a developmental scale stage to a modeled canopy provides a convenient way for the model user to visualize the "crop" which exists only in computer memory.

In many models, such as CERES-Wheat (Ritchie, 1985; Godwin and Vlek, 1985), WINTERWHEAT (Baker et al., 1985), and TAMW (Maas and Arkin, 1980 ), phenological status is reported by identifying

D.M. Harrell, Dep. of Agronomy, Univ. of Nebraska, Lincoln, NE 68583; W.W. Wilhelm, USDA-ARS, Dep. of Agronomy, Univ. of Nebraska, Lincoln, NE 68583; G.S. McMaster, USDAARS, Great Plains Systems Res., P.O. Box E, Fort Collins, CO 80522. Joint contribution of USDA-ARS and Nebraska Agric. Res. Div. Published as Journal Series no. 9961, Agric. Res. Div., Univ. of Nebraska. Received 21 May 1992. *Corresponding author.

Published in Agron. J. 85:758-763 (1993). major developmental stages (e.g., tillering, jointing, booting) rather than calculating a numerical scale value. Other models, such as ARCWHEAT (Weir et al., 1984), report development on a single scale, Haun (Haun, 1973) in this case. Few models report more than one scale; however, SHOOTGRO 2.0 (Wilhelm et al., 1990) and MODWHT (Waldman et al., 1990) report phenological results using the Haun, Feekes (Large, 1954), and Zadoks-Chang-Konzak (ZCK; Zadoks et al., 1974) scales.

The Feekes, Haun, and ZCK scales are the most widely used for wheat. The Feekes and ZCK scales are descriptive, nonlinear scales based on irregularly spaced phenological events from planting (ZCK) or emergence (Feekes) to plant maturity. The ZCK scale is essentially an expansion of the Feekes scale to provide more detail and wider geographic applicability. In contrast, the Haun scale is continuous and approximately linear in time and thermal units. It extends from emergence to anthesis. The Haun value at each phenological stage is not fixed but reflects the number of leaves produced on the main stem prior to the current time, thus it is strongly influenced by cultivar, planting date, management, and environmental conditions. These differences prevent a simple numeric correspondence among the three scales, particularly between the Haun scale and the other two. For this reason, conversion between scales requires knowledge of wheat phenological development and the scales themselves. The three scales differ in approach to stage designation and in level of detail. Consequently, they differ also in the applications to which they are best suited. Hence, it is likely that all three will continue in use and there is benefit in developing ways to convert and correlate the scales.

Because many agronomists may have only a passing acquaintance with developmental scales other than the one they consider most appropriate for their purposes, and wheat development models generally report results using no more than one scale, we identified a need for a computer program to aid in the conversion between developmental scales. Such a program could make it easier to interpret experimental and simulation results reported on an unfamiliar scale and to report results using more than one scale. The purpose of this paper is to describe the computer program, named SCALES, constructed to convert information on wheat development from emergence to maturity, among the Feekes, Haun, and ZCK scales.

\section{PROGRAM DESCRIPTION AND EXECUTION Overview}

SCALES evolved during the development of SHOOTGRO 2.0 , a model of vegetative growth and development of winter 
wheat (Wilhelm et al., 1990). Although the model is based on the Haun scale because of its applicability to the heat-unit/ phyllochron approach, SHOOTGRO 2.0 reports wheat development on all three scales through use of an algorithm that converts Haun values to the Feekes and ZCK scales. SCALES generalizes this approach to allow conversion from any of the three scales to the other two and extends the period covered to crop maturity. (SHOOTGRO 2.0 simulates winter wheat development and growth from planting through late boot only.)

The program is a dichotomous key, with the input scale value and additional user-supplied information determining the branches taken during execution of the key. Published descriptions (Haun, 1973; Large, 1954; Zadoks et al., 1974) and comparisons (Bauer et al., 1983; Landes and Porter, 1989) of the developmental scales were used to create the key. Development of the program required some interpretation of the scales not provided in the original descriptions.

\section{Design Considerations}

SCALES can be used in a variety of ways. The code is intended for use primarily as a module inserted into wheat development or growth models that provide the scale value(s) to be converted, as well as other required information. However, it is distributed as a stand-alone interactive program in which the user enters a value to convert along with additional information by answering questions prompted by the program. The conversion module can be uncoupled easily from the interactive module for incorporation into other programs.

Knowledge of the wheat plant developmental sequence was incorporated into SCALES to make the dichotomous key as efficient as possible. SCALES minimizes information requested of the user by requiring only information needed to convert a given value. For example, if SCALES has determined that the plant (canopy) is at anthesis, the program does not ask whether tillering is complete. SCALES also defers the most difficult questions. For example, it may be difficult for a user to determine if the flag leaf has appeared before flag leaf extension is nearly complete. SCALES does not request this information unless it has determined that jointing has occurred but flag leaf extension has not begun. At this point, the presence of the flag leaf is needed to distinguish Feekes Stage 8 from Stages 6 and 7.

SCALES retains as much information as each scale permits. This is accomplished by using the secondary stages of the Feekes and ZCK scales and by using three codes concurrently on the ZCK scale to reflect the number of main stem leaves and tillers throughout the development of the plant.

\section{Code}

SCALES is written as three modules: (i) keyboard input, (ii) dichotomous key, and (iii) screen output (Fig. 1). The most important of these is the dichotomous key, which is a series of IF-THEN-ELSE statements based primarily on the comparison of Bauer et al. (1983). It is connected to the keyboard input module through the use of COMMON memory to provide access to the input information. From the input value and answers to subsequent questions, the keyboard input module determines which of the approximately 20 questions to ask (Table 1).

\section{Assumptions and Limitations}

SCALES assumes that accurate information is provided. Although the interactive portion of the program performs nominal error checking to prevent input of values that are unquestionably out of range, the dichotomous key will not produce reliable results from conflicting inputs, nor will it identify unreliable results. A major source of potential problems is the reliance of the Haun scale on the number of leaves produced on the main stem rather than the number of leaves currently visible. If the user fails to account for older leaves that are senescent and may no longer be present, SCALES will produce Haun values that are too low.

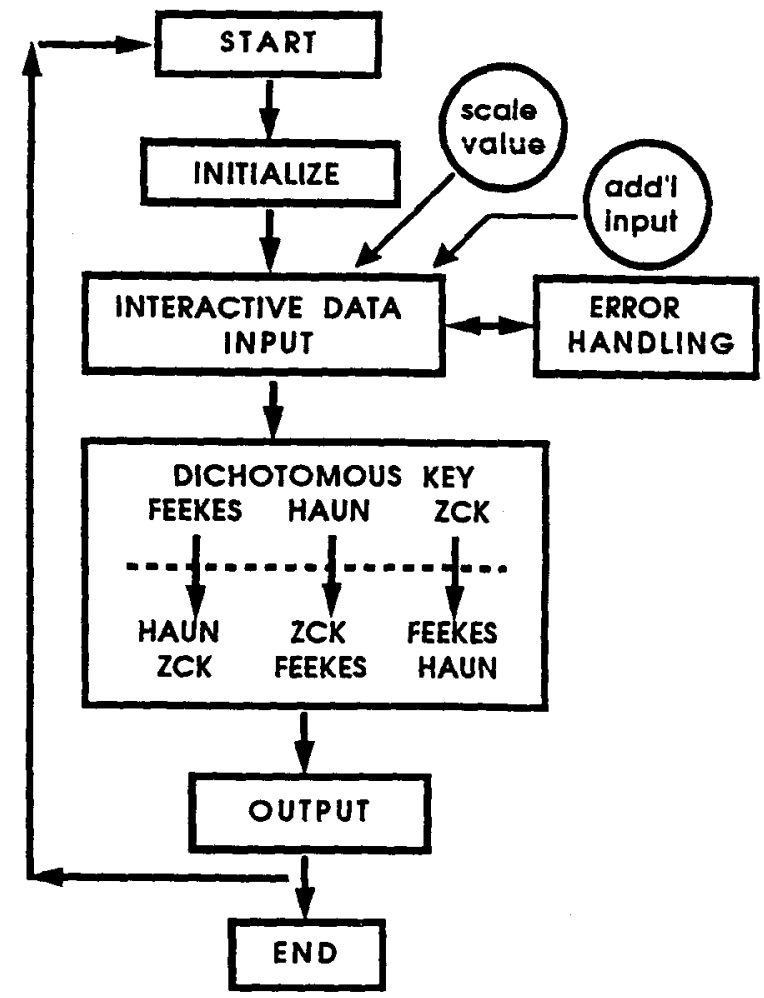

- Initialize Haun, Feekes, and ZCK values to 0

- Request scale value to convert

- Request additional information needed to convert value to other scales (see Table 1)

- Series of IF-THEN-ELSE statements

- Initial and conversion values printed to screen

Fig. 1. Components of SCALES program. 
Table 1. Questions asked of user. Only the information needed to convert the entered scale value is requested. SCALES asks Questions 1 and 2 for every conversion. Of the remaining questions, at most six are needed for any conversion. Following each question are letters indicating the input scales for which the question may be needed $(F=$ Feekes, $H=$ Haun, $Z=$ ZCK).

1. Are you entering Feekes, Haun, or ZCK data? $F, H, Z$

2. Enter the value you wish to convert. $F, H, Z$

3. How many fully expanded leaves has the main stem produced? $F, H, Z$

4. Will development be entered as two actual lengths or as an estimate of the ratio? $F, Z \dagger$

5a. Enter length of main stem leaf 1 , and

$5 b$. Enter maximum potential length of leaf 1 ; or

5c. Enter the relative development of main stem leaf $1 . F, Z$ (Repeat Question 4.)

6a. Enter length of youngest leaf, and

6b. Enter length of next-youngest leaf; or

6c. Enter the relative development of the youngest leaf. $F, Z$

7. How many tillers are present on the plant? $F, Z$

8. Is tillering complete? $H, Z$

9. Have the leaf sheaths begun to lengthen? $H$

10. Are leaf sheaths strongly erect? $H, Z$

11. How many nodes are visible on the main stem? $F, H$

12. Has the main stem flag leaf appeared? $H$

13. Is the main stem flag leaf fully expanded? $H$ (Repeat Question 4.)

14a. Enter length of flag leaf sheath, and

14b. Enter length of penultimate leaf; or

14c. Enter relative extension of flag leaf. $F, Z$

15. How far up into flag leaf sheath has head progressed (proportion from 0.0 to 1.0$)$ ? $F, Z$

16. Enter the number best describing the plant: (1) boots just visibly swollen, (2) boots fully swollen, (3) flag leaf sheaths beginning to open, or (4) awns visible or head escaping through split in sheath. $F$

17. Enter proportion of head extended beyond flag leaf collar. $F, Z$

18. Which stage is the plant in: (1) elongation of peduncle, (2) anthesis, or (3) ripening? $H$

(Repeat Question 4.)

19a. Enter length of peduncle, and

19b. Enter length of flag leaf; or

19 c. Enter relative extension of peduncle. $F, Z$

20. How far has anthesis progressed (proportion from 0.0 to 1.0)? $H$

21. How ripe is the grain: (1) milky ripe, (2) mealy ripe, (3) kernels hard, or (4) mature? $H$

$\lceil$ This question precedes questions $5,6,14$, and 19 , and determines which questions are asked ( $a$ and $b$, or $c$ ).

Some assumptions were needed where the original descriptions of the scales are ambiguous or open to interpretation. SCALES assumes that Feekes Stage 3, described as "tillers formed" (Large, 1954), is reached when tillering has been completed. In situations where tiller appearance continues after leaf sheaths begin to lengthen, SCALES will skip Feekes Stage 3. Also, Feekes Stage 7, described as "second node of stem formed; next to last leaf just visible" is defined in SCALES as "second node visible." This distinction is needed in situations where the two events are not simultaneous. Node detection was chosen as the stage indicator because it is impossible for an observer to determine (except in hindsight) whether the youngest leaf present on the main stem is the penultimate leaf.

The direction of the dichotomous key and the order of the IF tests are influenced by the decision to minimize the amount of information requested from the keyboard input module and to defer requests for information that might be difficult to provide. This results in a key that is not in chronological order. However, the order in which questions are asked does not affect the results produced from accurate inputs.
SCALES begins with seedling emergence because the Haun and Feekes scales do not consider germination and seedling elongation. The ZCK values less than $\mathbf{1 0}$ result in Haun and Feekes values of 0 . Although the Haun scale ends at anthesis, SCALES continues beyond that point, assigning the Haun value for anthesis to all later stages.

The program uses Feekes values in decimal format. This requires that Feekes values such as 10.5 .1 be supplied to the program as 10.51 .

\section{RESULTS AND DISCUSSION}

SCALES output agrees well with the correlations of Bauer et al. (1983) on which it was based (Table 2). The results are valid within the limits of the program's purpose and design. As indicated earlier, SCALES was not designed to resolve conflicting inputs nor provide best estimates when required inputs are not available.

The number of questions the user must answer, in addition to providing the scale value to convert, ranges from 0 to 6 . Generally, conversions of Haun values to the other scales require the most additional inputs (range 4-6, compared with 2-6 for Feekes input and 0-6 for ZCK). This occurs because the Haun value alone does not provide much information about plant development other than the number of developed leaves. In contrast, converting a ZCK value for a plant that has reached anthesis (third ZCK code value 60 or greater) requires no further questions if the plant has produced fewer than nine main stem leaves (i.e., first ZCK code value is less than 19 and indicates the exact number of leaves). Table 3 is a sample terminal session with SCALES.

Stage numbers may be skipped as a plant develops because of variability in the development of the wheat plant or inaccurate inputs. As an example of the first case, if the flag leaf appeared before the third node of the main stem appeared, the ZCK value would skip from 32 to 37 . An example of the latter case would result if an inexperienced observer indicated that the flag leaf was fully expanded before the first node became visible (jointing), causing SCALES to skip Feekes Stages 6, 7, and 8.

Although Table 2 shows distinct boundaries between major developmental stages, in reality many of the stages overlap. This rarely causes problems in the SCALES conversion because actual scale numbers and measurable parameters are used whenever possible. Qualitative characteristics (e.g., Is tillering complete? Has the main stem flag leaf appeared?) are used primarily to make distinctions within the major stages. The most notable exception is Question 18 (Table 1), asked during conversion of Haun values. This question is required to distinguish anthesis from ripening since both have the highest value available on the Haun scale.

The final stage on the Haun scale (culm, or peduncle, elongation) is problematic. Haun (1973) specifically excludes the phases of flowering and ripening and assigns peduncle elongation to a separate stage with scale numbers ranging from $F+3.0$ to $F+4.0$ ( $F$ is the number of the flag leaf). The Feekes and ZCK scales do not include peduncle elongation as a separate stage. Peduncle elongation during booting, heading, and into anthesis supports the Feekes and ZCK approach. Landes and Porter (1989) suggest that the Haun scale is not generally useful after flag leaf extension, since the developmental criteria are species dependent. They do not include pe- 
Table 2. Relationships among wheat growth stages produced by SCALES.

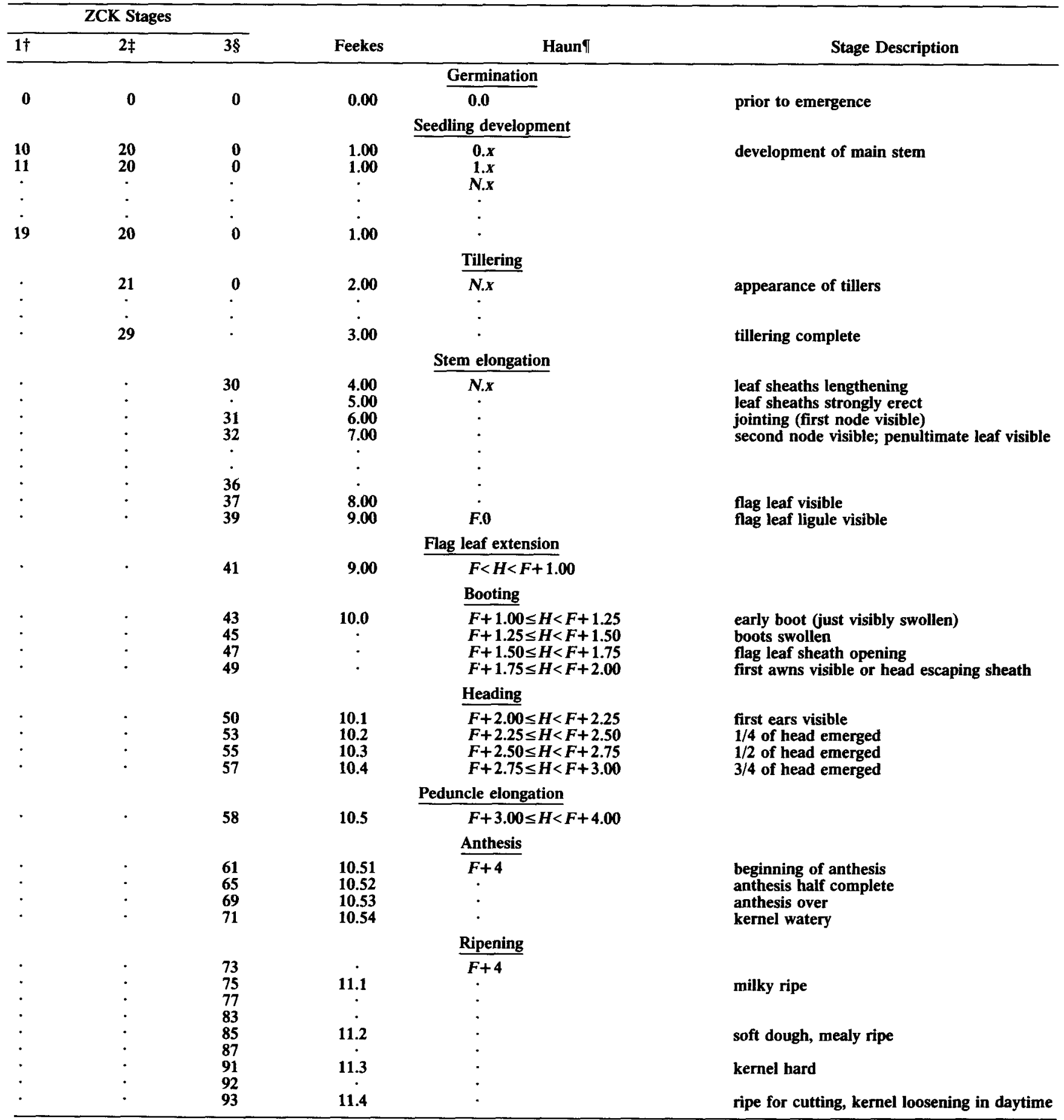

First number on ZCK scale is 10 plus the number of fully expanded leaves on the main stem; maximum value is 19. Note that main stem leaf appearance is not complete by the time tillering begins and may extend into the late stem elongation phase.

¥ Second number on ZCK scale is $\mathbf{2 0}$ plus the number of tillers on the plant; maximum value is 29 . Note that tiller production may continue into the stem elongation phase.

$\$$ Values of third number on ZCK scale ranging from 30 to 36 reflect the number of visible nodes on main stem (0-6). The plant may reach Stage 37 after fewer than six nodes have become visible, skipping intermediate values.

IFrom emergence until flag leaf is fully expanded, Haun is calculated as number of fully expanded leaves $(N)$ on main stem plus relative development of youngest leaf on main stem. After the flag leaf is completely expanded, exact Haun value lies within the range shown and is calculated from user's responses to various questions about the progress of each stage. ( $H=$ Haun value; $F=$ leaf number of main stem flag leaf.)

duncle elongation as a separate stage. Bauer et al. (1983) describe anthesis as terminating the last phase on the Haun scale (culm elongation), but show both peduncle elongation and anthesis occurring during the final phase
(Table 5; Bauer et al., 1983). SCALES addresses this problem by allowing the user to select any of three developmental stages (peduncle elongation, anthesis, or ripening) when converting a Haun value of $F+3.0$ or 
Table 3. Sample terminal session with SCALES. Program questions are shown in normal type; user responses in bold italic type. User entered three values for conversion, first a Haun value, then a Feekes value, and finally a ZCK value.

Are you entering Haun, Feekes, or ZCK data? (h, f, or $\mathrm{z}$ )

Enter the Haun stage. (0.0 to 25.0)

14.0

How many tillers are present on the plant? (0 to 20)

How many nodes are visible on the main stem? (0 to 10)

4

Is the main stem flag leaf fully expanded? $(\mathrm{y}$ or $\mathrm{n}$ )

y

How many leaves has the main stem produced? ( 0 to 20 )

10

Which stage is the plant in?

(1) elongation of peduncle (spike extension)

(2) anthesis

$(1,2$, or 3$)$

(3) ripening

How far has anthesis progressed?

(fraction complete: 0.0 to 1.0 )

0.6

Interactive input complete.

Haun value entered: 14.00

Equivalent Feekes stage: 10.52

Equivalent ZCK scale numbers: 192565

Do you wish to convert another value? ( $y$ or $n$ )

y

Are you entering Haun, Feekes, or ZCK data? (h, f, or $z$ )

Enter the Feekes stage. (0.0 to 11.4)

10.

How many fully expanded leaves has the main stem produced? (0 to 20)

How many tillers are present on the plant? (0 to 20)

How far up into flag leaf sheath has head progressed? (proportion from 0.0 to 1.0 )

0.75

Enter the number best describing the plant:

(1) boots just visibly swollen

(2) boots fully swollen

(3) flag leaf sheaths beginning to open

(4) awns visible or head escaping through split in sheath (1 to 4)

Interactive input complete.

Feekes value entered: 10.00

Equivalent Haun stage: $\mathbf{1 0 . 7 5}$

Equivalent ZCK scale numbers: 192647

Do you wish to convert another value? ( $y$ or $n$ )

Are you entering Haun, Feekes, or ZCK data? (h, f, or $\mathrm{z}$ )

z
Table 3. Continued

Enter the first of three ZCK stage numbers (related to number of leaves produced by main stem). (0, or 10 to 19$)$

16

Enter the second of three ZCK stage numbers (related to number of tillers present on plant). (0, or 20 to 29)

Enter the third of three ZCK stage numbers (related to main stem development). (0, or 30 to 95 )

Will leaf development be entered as

(1) actual lengths of 2 youngest leaves, or

(2) relative development of youngest leaf? (1 or 2)

Enter length of the youngest leaf (mm). (0.0 to 500.0$)$

150.

Enter length of the second youngest leaf $(\mathrm{mm}) .(0.0$ to 500.0$)$

260.

Is tillering complete? $(\mathbf{y}$ or $\mathbf{n})$

n

Interactive input complete.

Zadoks-Chang-Konzak values entered: 16220

Equivalent Haun stage: 6.58

Equivalent Feekes stage: 2.00

Do you wish to convert another value? ( $y$ or $\mathbf{n}$ )

Program has terminated normally.

higher. If peduncle elongation is selected when the Haun is less than $F+4.0$, the result is a Feekes value of 10.5 and a ZCK value (third code) of 58, both of which indicate that heading is complete. However, if anthesis is chosen, appropriate Feekes and ZCK values will result, consistent with Bauer et al. (1983).

The conversion module of SCALES is a useful tool for computer simulations of wheat development and growth. It can provide a more detailed report of phenological stage than most current models present. It also facilitates comparisons among models that use different developmental scales.

\section{SPECIFICATIONS, DOCUMENTATION, AND AVAILABILITY}

SCALES is written in ANSI standard FORTRAN 77. The program is designed for IBM-compatible personal computers running MS-DOS or PC-DOS version 3.3 or higher. ${ }^{1}$ It requires $61 \mathrm{~K}$ of RAM. A hard disk and math co-processor are not required. SCALES has been tested on an IBM mainframe running CMS and a Hewlett-Packard running UNIX.

SCALES is available from the USDA-ARS Soil and Water Conservation Research Unit, 119 Keim Hall, University of Nebraska, Lincoln, NE, 68583-0934. The source code, executable program, and documentation are distributed on diskette. Please indicate the type of ma-

\footnotetext{
${ }^{1}$ Mention of a trademark or proprietary product does not constitute an endorsement by the authors, the University of Nebraska, or the USDA.
} 
chine that will be used to run SCALES and the type and size of diskette needed (5.25-inch or 3.5-inch; high or standard density).

\section{REFERENCES}

Baker, D.N., F.D. Whisler, W.J. Parton, E.L. Klepper, C.V. Cole, W.O. Willis, D.E. Smika, A.L. Black, and A. Bauer. 1985. The development of WINTER WHEAT: A physical physiological process model. p. 176-187. In W.O. Willis (coordinator), ARS Wheat Yield Project, USDA, Agricultural Research Service ARS-38. U.S. Gov. Print. Office, Washington, DC.

Bauer, A., D. Smika, and A. Black. 1983. Correlation of five wheat growth stage scales used in the Great Plains. USDA, Agricultural Research Service, AAT-NC-7. Peoria, IL

Godwin, D.C., and P.L.G. Vlek. 1985. Simulation of nitrogen dynamics in wheat cropping systems. p. 311-332. In W. Day and R.K. Atkin (ed.) Wheat growth and modeling. Plenum Press, New York

Haun, J.R. 1973. Visual quantification of wheat development. Agron. J. 65:116-119.

Landes, A., and J.R. Porter. 1989. Comparison of scales used for categorising the development of wheat, barley, rye and oats. Ann. Appl. Biol. 115:343-360.
Large, E.C. 1954. Growth stages in cereals. Plant Pathol. 3:128129.

Maas, S.J., and G.F. Arkin. 1980. TAMW: A wheat growth and development simulation model. Texas A\&M Univ., Texas Agric. Exp. Stn., Program and Model Documentation no. 80-3.

Ritchie, J.T. 1985. A user-oriented model of the soil water balance in wheat. p. 293-305. In W. Day and R.K. Atkin (ed.) Wheat growth and modeling. Plenum Press, New York.

Waldman, S.E., R.W. Rickman, E.L. Klepper, and A. Sombrero. 1990. Field validation of MODWHT a modular wheat model. p. 24. In Agronomy abstracts. ASA, Madison, WI.

Weir, A.H., P.L. Bragg, J.R. Porter, and J.H. Rayner. 1984. A winter wheat crop simulation model without water or nutrient limitations. J. Agric. Sci. (Camb.) 102:371-382.

Wilhelm, W.W., G.S. McMaster, B. Klepper, and R.W. Rickman. 1990. A vegetative development and growth model for wheat. p. 34-35. In R.C. Munchow and J.A. Bellamy (ed.) Climatic risk in crop production; Models and management for the semi-arid tropics and subtropics. Proc. Int. Symp., Brisbane, Queensland, Australia. 2-6 July. CSIRO, Brisbane.

Zadoks, J.C., T.T. Chang, and C.F. Konzak. 1974. A decimal code for the growth stages of cereals. Weed Res. 14:415421 . 